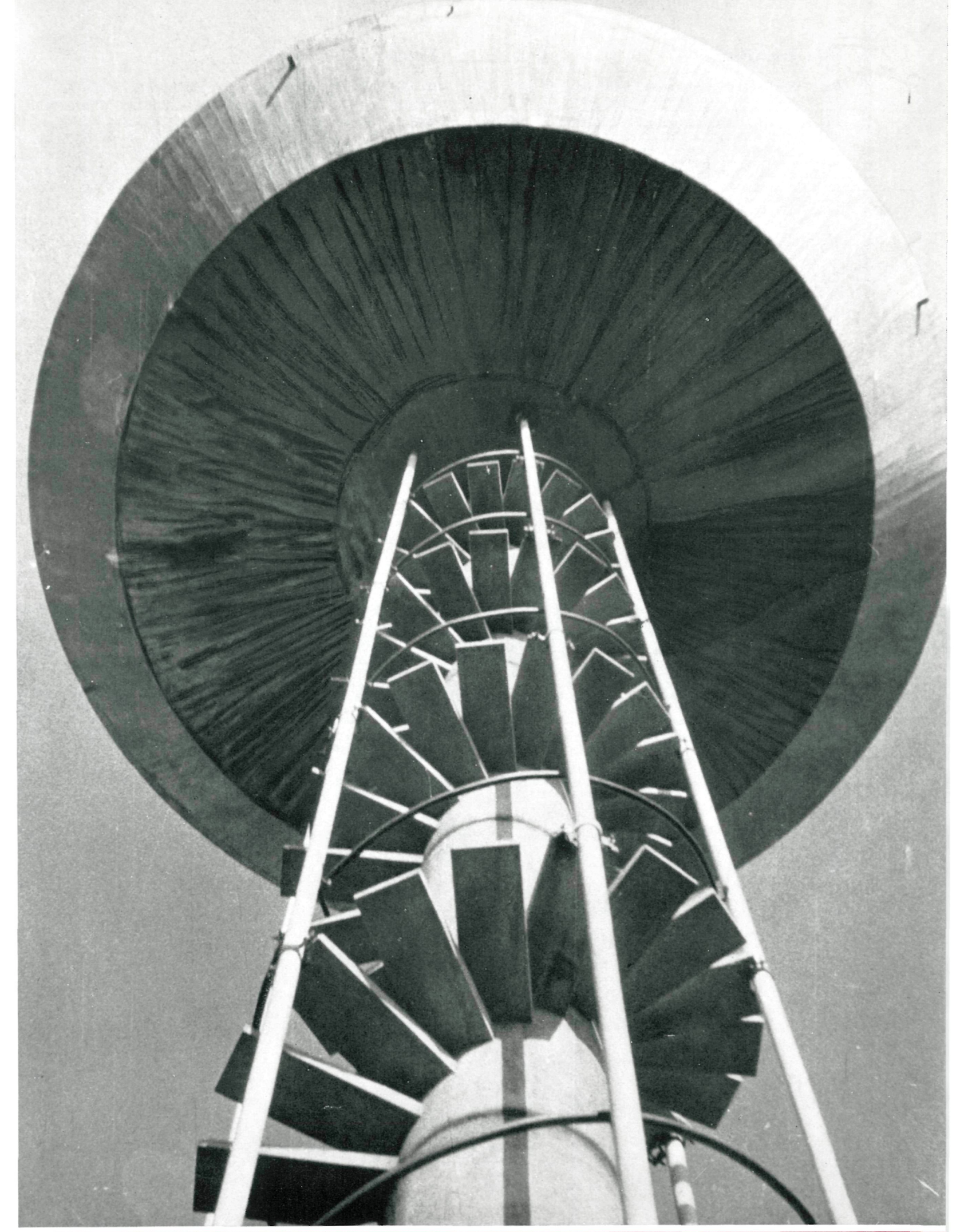

\title{
depósito elevado
}

\section{de hormigón armado}




\section{simopsis}

Para abastecer de agua a un gran conjunto industrial que construye la $\mathrm{Em}$ presa S. A. D. E. cerca de Buenos Aire (Argentina), se ha construido un depó sito elevado, de $80 \mathrm{~m}^{3}$ de capacidad $21 \mathrm{~m}$ de altura. La cubeta, de hormigón armado, tiene la forma de una copa aplanada de planta circular y se ha subdividido en cuatro compartimientos comunicados dos a dos, simetricos respecto al eje vertical, con objeto de conseroperaciones de carga y vaciado $L$ particularidad de este depósito consiste en su método eonstructivo y en los proce dimientos de prefabricación empleados. El fuste o soporte único central es un cilindro hueco, formado por trozos prefabricados, de 1,60 $\mathrm{m}$ de longitud. El de base se apoya sobre una losa circular -que descansa sobre cuatro pilotes cilíndricos, de hormigón, prefabricados de $15 \mathrm{~m}$ de longitud-debajo de la cual se han instalado las válvulas de paso. E acceso a la parte superior se realiza por medio de una escalera en espiral, cuyo peldaños se apoyan en el fuste cilíndrico. Para mayor estabilidad entre eletálicas de han , de al a provechado, además, para apoyar de vuelco creado por los efectos de viento y sobrecargas móviles se contrarresta por medio de seis vigas radiales, de $5 \mathrm{~m}$ de Iongitud.

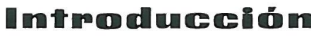

El complejo industrial construido por S. A. D. E. (Sociedad Argentina de Electrificación, S. A ) en los alrededores del Gran Buenos Aires, ocupa unos $50.000 \mathrm{~m}^{2}$, de los cuales se han reservado $10.000 \mathrm{~m}^{2}$ para talleres, $5.000 \mathrm{~m}^{2}$ para oficinas, vestuarios y comedores, etc., y $2.500 \mathrm{~m}^{2}$ para calles y estacionamiento. Este conjunto se destinará a talleres para S. A D. E. y para Electromecánica Argentina, S. A., empresa dedicada a la fabricación de instrumental eléctrico. El abastecimiento de agua y reserva contra incendios requiere un volumen de $80 \mathrm{~m}^{3}$, con una presión de dos atmósferas, a cuyos efectos se decidió la construcción de un tanque elevado de hormigón armado.

\section{Descrmipción gemencan del depósito}

Por razones aerodinámicas y constructivas, se adoptó la forma de un plato de bordes agudos, como muestran los planos y fotografías adjuntas. Dada la necesidad de dividir el tanque en com partimientos para usos distintos del agua en el depósito, se proyectaron dos diafragmas cruzados, que forman cuatro recipientes independientes. Los compartimientos opuestos se comunican así con las tuberías de desagüe, con lo cual se garantiza el equilibrio de la carga.

Vista general.

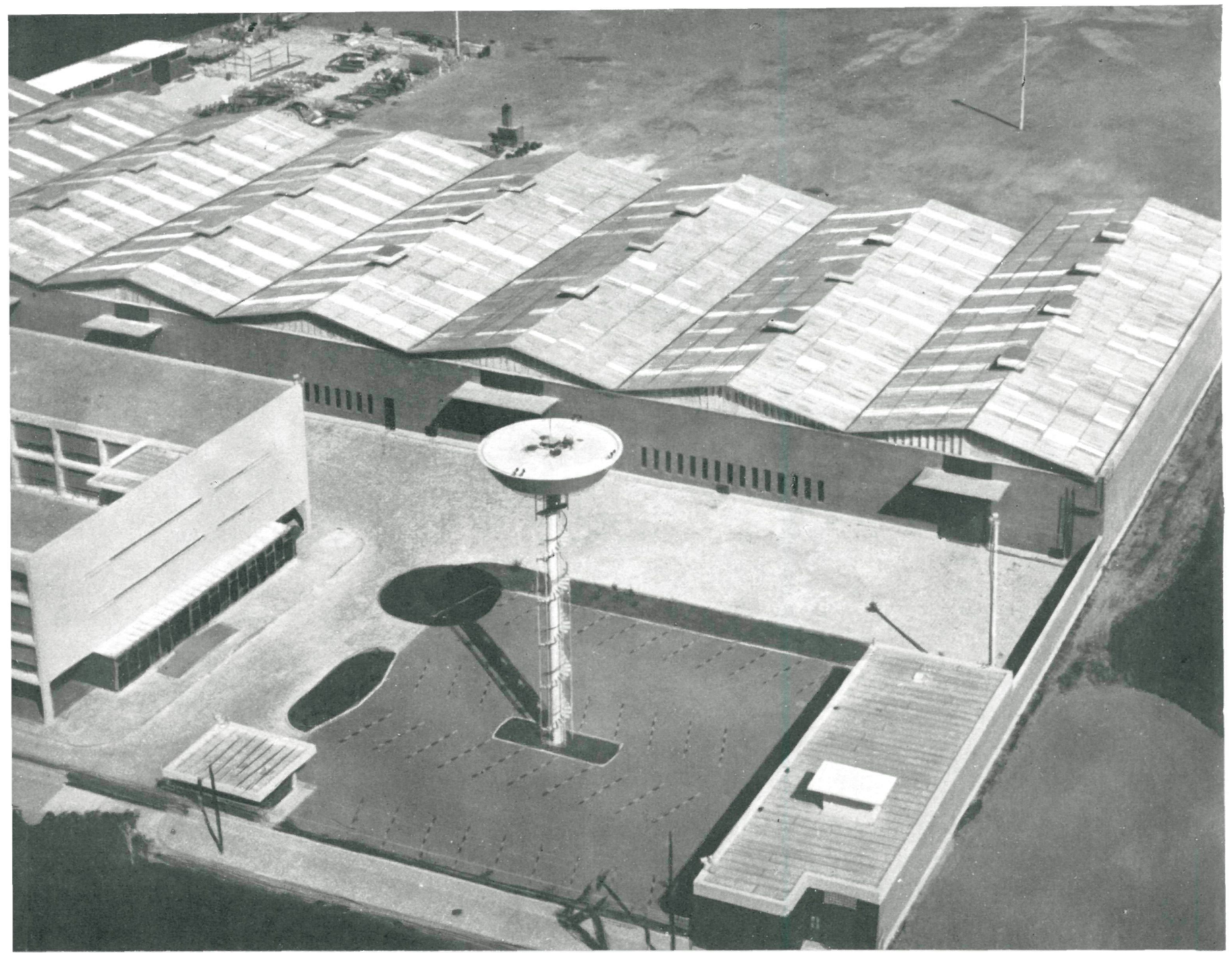




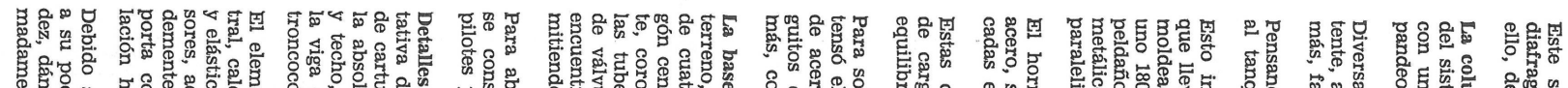

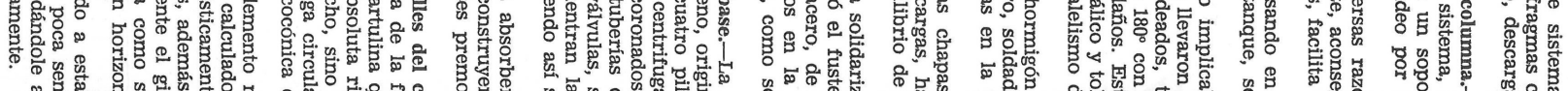

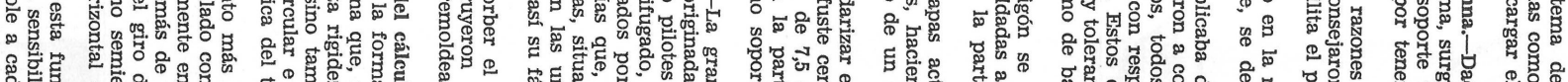

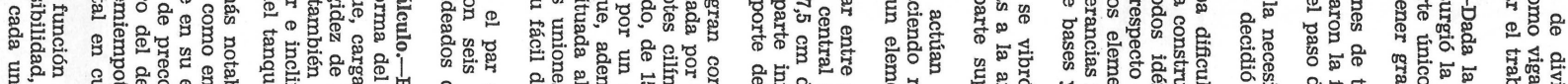

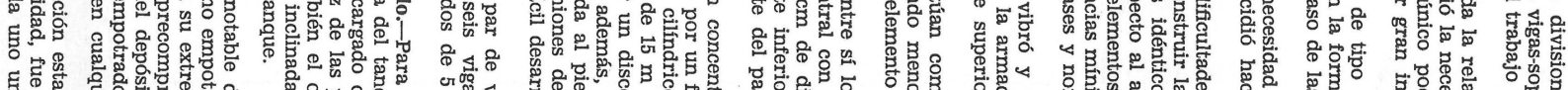

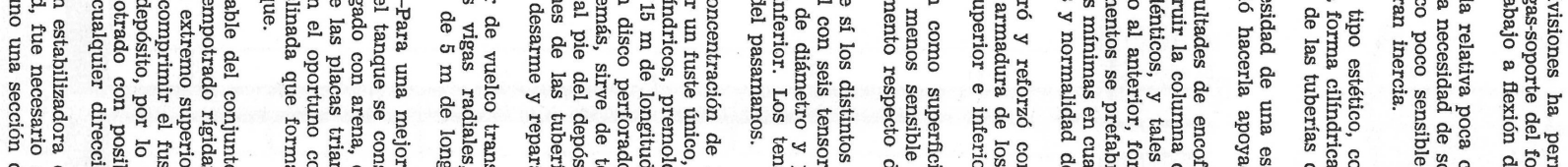
क

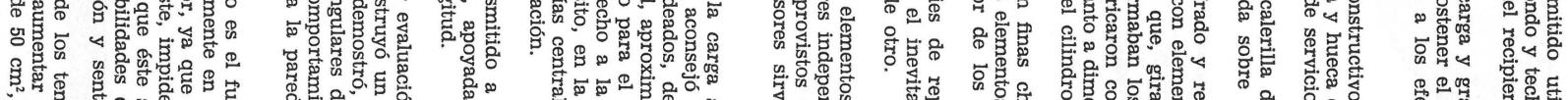

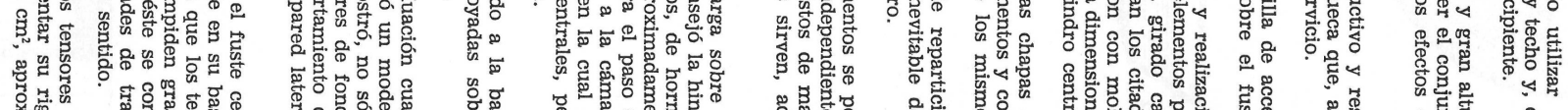

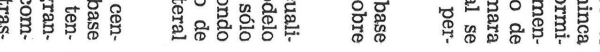
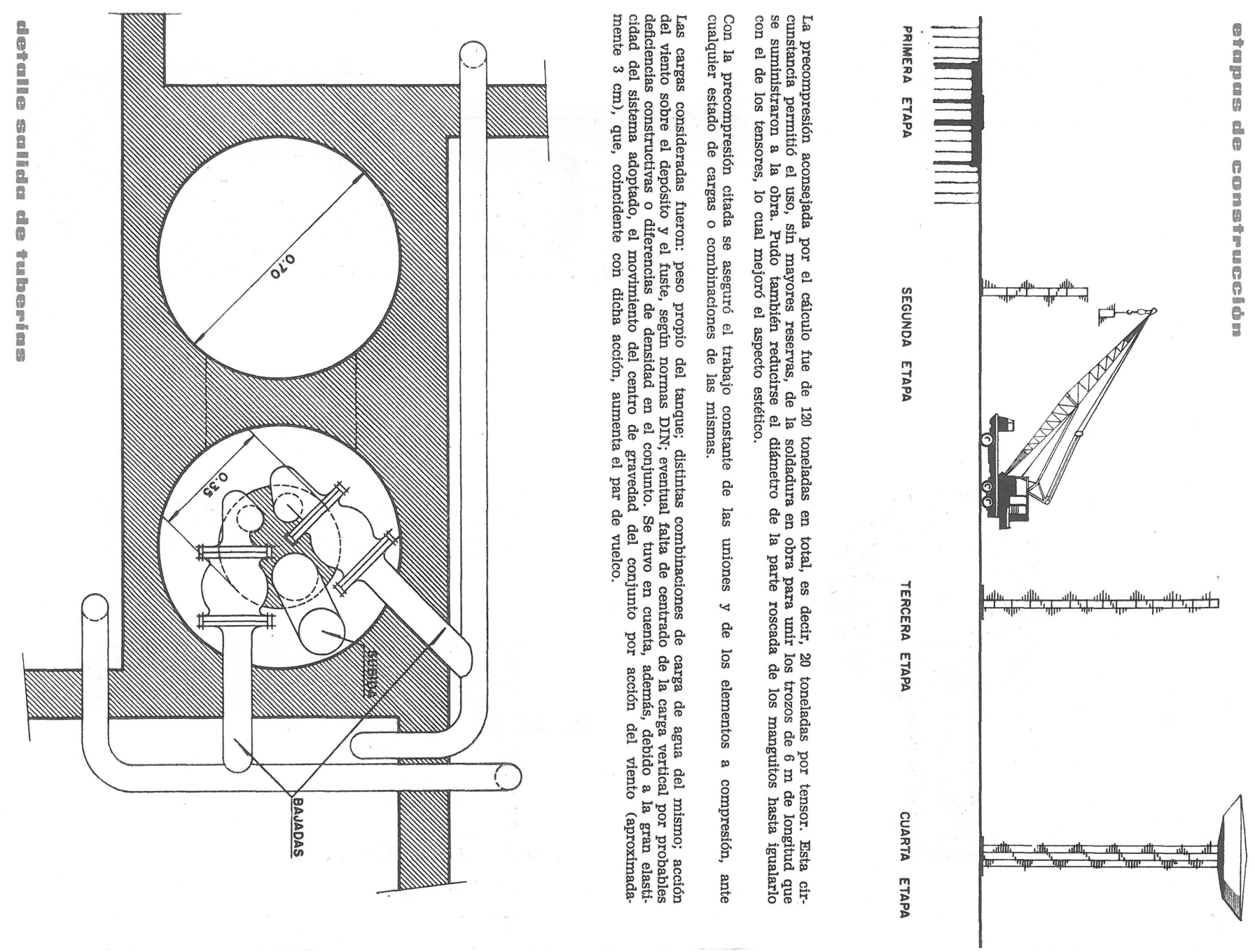

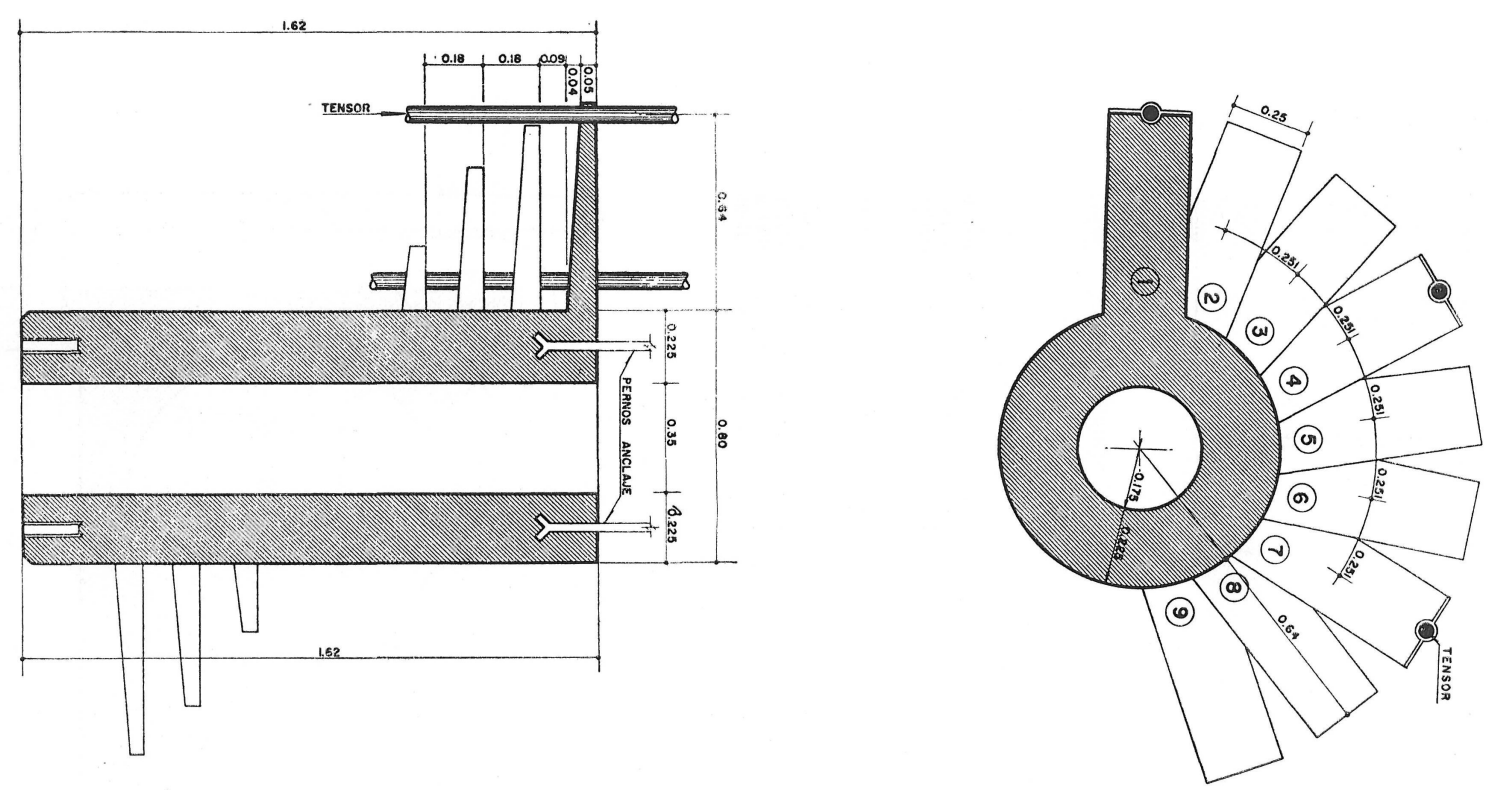

를
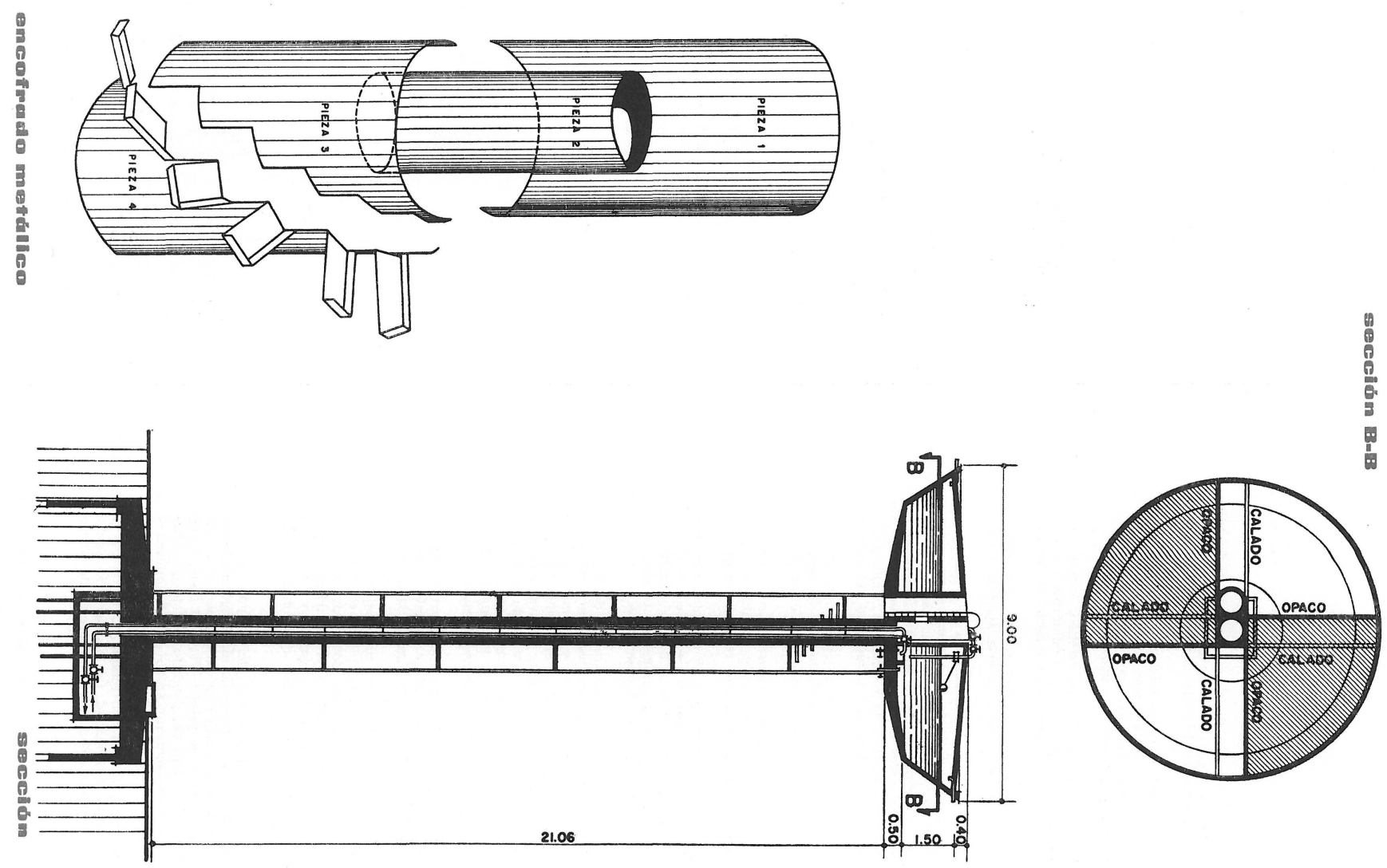

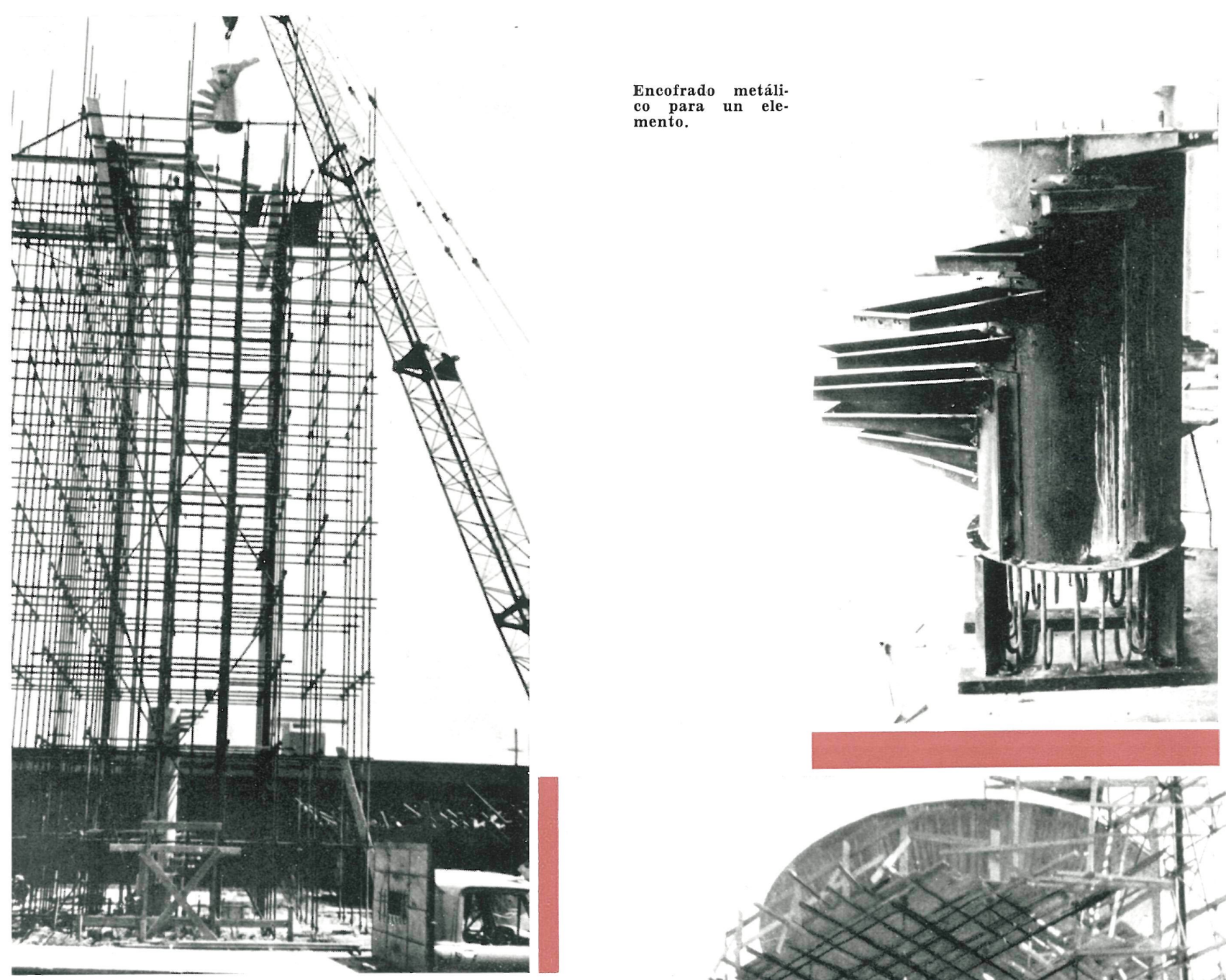

Construcción del fuste.
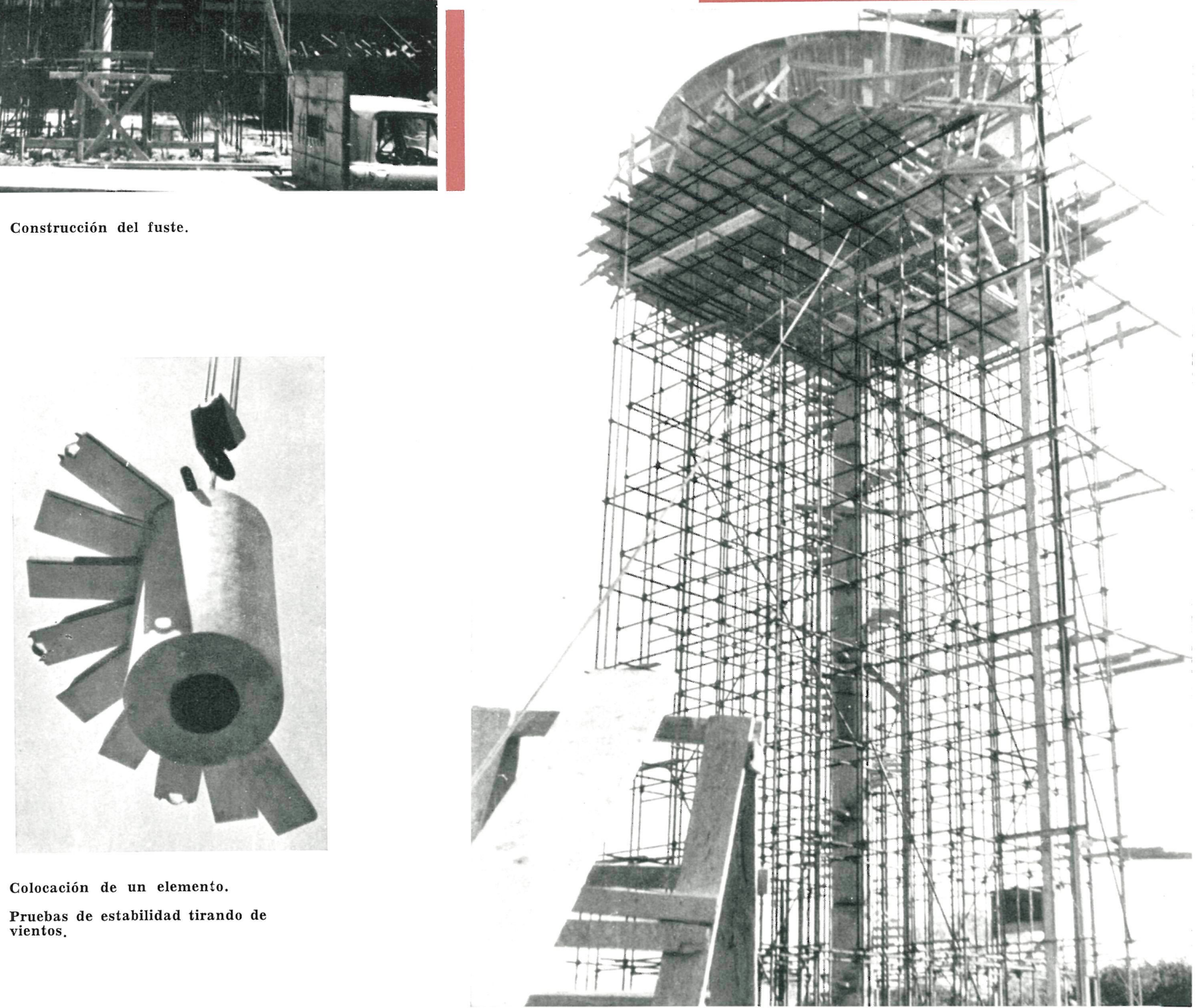

Colocación de un elemento.

Pruebas de estabilidad tirando de vientos. 
De esta forma se llegó a un fuste de $80 \mathrm{~cm}$ de diámetro exterior, $35 \mathrm{~cm}$ de diámetro interior y $20 \mathrm{~m}$ de altura, que trabaja siempre a compresión y tiene, en la peor combinación de cargas, una tensión de $110 \mathrm{~kg} / \mathrm{cm}^{2}$ en el borde. La tensión real de trabajo máxima es de $90 \mathrm{~kg} / \mathrm{cm}^{2}$, aproximadamente, lo que permitió operar con hormigones normales en la prefabricación de los elementos.

Para aumentar la seguridad del conjunto disminuyendo la luz de pandeo, el primero y último elemento se empotraron en la base y depósito, respectivamente. Para el cálculo se siguieron las normas locales vigentes, aunque la verificación de los resultados por cálculo plástico demostró una seguridad mayor que la exigida.

Montaje del fuste y construcción del depósito.-Los elementos se montaron con una pluma móvil, de $30 \mathrm{~m}$ de longitud, durante dos jornadas normales de trabajo, incluyendo la colocación de los elementos, nivelación de los mismos y punteado por soldadura de las chapas de apoyo, una vez obtenido el centrado perfecto.

La operación fue más fácil gracias a la escalera helicoidal y al andamiaje tubular desmontable que estaba previsto para soportar el encofrado del depósito, que se realizó con tolerancias de 2 ó $3 \mathrm{~mm}$. Se empleó hormigón de fraguado rápido y se desencofró a los doce días.

Desde el comienzo del montaje del fuste hasta la puesta en servicio del tanque transcurrieron ochenta días, aproximadamente.

Postensado.-Una vez desencofrado el depósito se procedió a tesar las armaduras por medio de sus manguitos, simultáneamente y por parejas opuestas. La tensión fue medida por el alargamiento provocado en el tensor (del orden de los $6 \mathrm{~mm}$ ), para lo cual se hicieron previamente varias determinaciones, en laboratorio, del módulo de elasticidad del material empleado. La medición del par necesario para el giro de los manguitos permitió, además, la verificación de la tensión obtenida por aplicación del principio de los trabajos virtuales.

El tensado a tanque vacío produce una compensación con la carga de agua del mismo, puesto que sus efectos no se superponen.

Para prevenirse contra la pérdida de tensión debida a la dilatación térmica, se los protegió con pintura reflectante mezclada con amianto.

Se prevé, además, la verificación en el tiempo de fluencia del fuste, para ajustar, si es necesario, los tensores.

Con el fin de evitar la penetración de humedad por las juntas entre elementos, se soldaron posteriormente las dos chapas, aunque la fricción entre las mismas hubiera sido suficiente para transmitir el pequeño esfuerzo cortante.

La prueba.-Para verificar el resultado de la construcción se realizó un ensayo consistente en tirar con un cable amarrado al depósito del tanque y a $45^{\circ}$, aproximadamente, para provocar un estado de solicitación equivalente al de cálculo.

En dicho cable se aplicó un esfuerzo de 3 toneladas (medidas con dinamómetro a resorte)-equivalentes a una fuerza de 2 toneladas aplicadas horizontalmente y a nivel del depósito-, las cuales produjeron un desplazamiento horizontal de $3 \mathrm{~cm}$ del "centro del tanque. Este desplazamiento se midió con plomada interior al fuste, observando una plena recuperación elástica a la descarga.

El proyecto, cálculo, dirección técnica, construcción y montaje, estuvo a cargo de la Oficina de Obras Civiles de S. A. D. E.-Sociedad Argentina de Electrificación, S. A.-, bajo la dirección general del Ingeniero Jorge W. Magaldi. El estudio de suelos fue realizado por el Ingeniero Rogelio Héctor Rodríguez Vázquez, asesor de la Sociedad en esta especialidad.

La construcción de los pilotes y elementos premoldeados fue efectuada por S. C. A. C. (Sociedad Cementos Armados Centrifugados, S. A.).

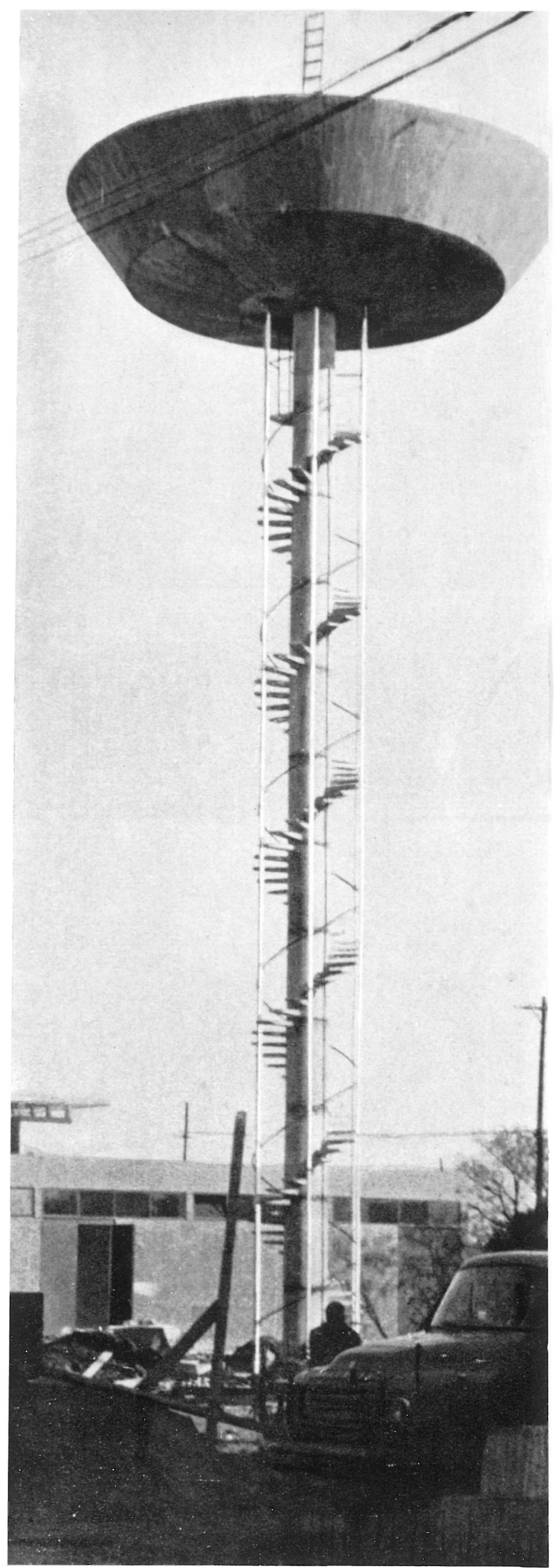




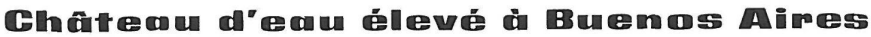

S. A. D. E.

Pour le ravitaillement en eau du grand ensemble industriel que construit l'entreprise S. A. D. E. près de Buenos Aires (Argentine), on a construit un château d'eau de $21 \mathrm{~m}$ de haut et d'une capacité de $80 \mathrm{~m}^{3}$.

La réservoir, en béton armé, a la forme d'une coupe très aplatie circulaire. Il a été divisé en quatre compartiments qui communiquent deux à deux et symétriques par rapport à l'axe vertical, afin de conserver l'équilibre de charges pendant les opérations de charge et de vidange.

Les particularités de ce château d'eau ont été sa méthode de construction et les procédés de préfabrication employés.

Le fût ou support unique central est un cylindre creux, formé par des éléments préfabriqués, de $1 \mathrm{~m} 60 \mathrm{de}$ longueur. La

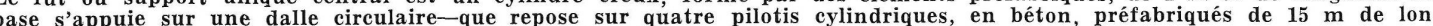
gueur-au-dessous de laquelle les vannes ont été installées.

L'accès à la partie supérieure se réalise au moyen d'un escalier en spirale dont les marches s'appuient sur le fût cylinmètre, qui servent, en même temps, d'appui à la rampe de l'escalier.

Le couple de renversement créé par les effets du vent es les surcharges mobiles est contrecarré à l'aide de six poutres radiales, de $5 \mathrm{~m}$ de longueur.

\section{Elevoned Trumk in Buemos Ainges}

S. A. D. E.

The firm S, A D. E., near Buenos Aires (Argentina), has built an elevated tank, with a capacity of $80 \mathrm{~m}^{3}$, at $21 \mathrm{~m}$ height, to supply water to its large industrial installations.

The tank is of reinforced concrete. It is shaped as a circular, rather shallow cup, which is subdivided into four compartments, connected in symmetrical pairs with respect to the central axis. This ensures a weight balance when it is filled and emptied.

This tank is of interest because of its method of construction, and the prefabrication procedure adopted.

The supporting stem is a hollow cylinder, made of prefabricated sections, each $1.60 \mathrm{~m}$ long. At the bottom there is a circular slab, which rests on four cylindrical concrete, prefabricated piles, each $15 \mathrm{~m}$ long. The system of control valves is placed below the slab.

Ascent to the top part is by means of a spiral staircase, whose steps are attached to the stem. To increase its stability, ix metal rods, $7.5 \mathrm{~cm}$ in diameter, have been added to the cylindrical stem, and the handrail for the steps has been conveniently attached to these.

The side thrusts due to wind forces acting on the tank are withstood by means of six radial beams, $5 \mathrm{~m} \mathrm{in} \mathrm{length.}$

\section{Hochloelhäitern in Buenos Aimes}

S. A. D. E.

Um einen grossen Industriekomplex, der von der Firma S. A. D. E. in der Nähe von Buenos Aires (Argentinien) gebaut wird, mit Wasser zu versorgen, wurde ein Hochbehälter von $83 \mathrm{~m}^{3}$ Fassungsvermögen und $21 \mathrm{~m}$ Höhe gebaut.

Er besteht aus Stahlbeton und hat die Form einer abgeflachten Tasse, die in 4 Quadranten eingeteilt ist, von denen je zwei symmetrisch zur senkrechten Achse miteinander verbunden sind, um das Gleichgewicht beim Füllen und Leeren aufrecht zu erhalten.

Die Eigenart dieses Hochbehälters besteht in seiner Baumethode und dem angewandten Vorfertigungsverfahren.

Die Stütze ist ein aus vorgefertigten Teilen hergestellter Hohlzylinder von 1,60 m Länge, der mit Hilfe einer kreisförmigen Platte auf vier zylindrischen, aus Beton vorgefertigten $15 \mathrm{~m}$ langen Pfählen aufliegt, unter denen man die Durchgangs ventile angebracht hat.

Mittels einer Spindeltreppe an der Säule gelangt man in den oberen Teil des Behälters. Aus Stabilitätsgründen hat man 6 Metallstangen von $7,5 \mathrm{~cm}$ Durchmesser vorgesehen, die gleichzeitig als Handgeländer für die Spindeltreppe dienen.

Das durch den Wind hervorgerufene Kippmoment und die Überbelastungen werden durch 6 radial verlaufende Träger von $5 \mathrm{~m}$ Länge aufgefangen. 